\title{
SPECTRAL PROPERTIES OF DEGENERATE ELLIPTIC OPERATORS WITH MATRIX COEFFICIENTS
}

\author{
M.G. GADOEV, S.A. ISKHOKOV
}

\begin{abstract}
In the work we study some spectral properties of the non-self-adjoint operator $A$ in the space $\mathcal{H}^{l}=L_{2}(0,1)^{l}$ associated with a noncoercive sesquilinear form. We address the issues on completeness of a system of root vector-functions for operator $A$ in $\mathcal{H}^{l}$, description of the domain of operator $A$, estimating resolvent of operator $A$ and asymptotic distribution of eigenvalues of operator $A$.
\end{abstract}

Keywords: elliptic differential operators, resolvent of operator, distribution of eigenvalues, system of root vector-functions.

Mathematics Subject Classification: 34L20, 34L10, 47E05, 47A10, 46E35

\section{INTRODUCTION}

The present paper is a continuation of work [1]. We study some spectral properties of a certain class of degenerate elliptic non-self-adjoint operators $A$ in the space $\mathcal{H}^{l}=L_{2}(0,1)^{l}$; the operators are associated with non-coercive sesquilinear forms. We also consider the issues on the completeness of the system of root vector-functions for an operator $A$ in $\mathcal{H}^{l}$, a description of the domain of an operator $A$, estimates for the resolvent of an operator $A$, asymptotic distribution of eigenvalues of an operator $A$.

Spectral asymptotics for degenerate elliptic operators far from being self-adjoint were studied in works [2-7] in the situation when the eigenvalues of the operator split into two series, one being located outside the angle $|\arg z| \leqslant \varphi, \varphi<\pi$, while the other was accumulating to the ray $R_{+}=(0,+\infty)$. This paper, as [1], is related to works $[2,3,7]$. The most general results were obtained in [7], where it was assumed that the leading coefficient of the operator $A$ satisfies

$$
a(t) \equiv a_{m m}(t) \in C^{m}\left([0,1] ; E n d \mathbb{C}^{l}\right)
$$

and has different simple eigenvalues for each $t \in[0,1]$.

Instead of $(0.1)$ we just assume $a(t) \in C\left([0,1] ; E n d \mathbb{C}^{l}\right)$.

\section{Formulation of MAin Results}

1. An operator $A$ acting a Hilbert space $H$ will be called far from being self-adjoint if it can not be reduced to the form

$$
A=B(E+D), \quad B=B^{*}, \quad D \in \sigma_{\infty}(H) .
$$

Hereinafter the symbol $\sigma_{\infty}(H)$ indicates the class of linear completely continuous operators in $H, B^{*}$ is the adjoint operator for $B$.

Spectral properties of elliptic differential and pseudodifferential operators close to self-adjoint, i.e., which can be reduced to (1.1), are studied well enough, see [8,9]. Also in details there were studied spectral properties of elliptic differential operators (DO) and pseudodifferential operators (PDO) far from being self-adjoint in the case when the are defined on a compact manifold without the boundary (see $[7,10-12]$ and the references therein). In the case of

M.G. Gadoev, S.A. Iskhokov, Spectral properties of Degenerate elliptic operators with MATRIX COEFFICIENTS.

(c) Gadoev M.G., Iskhokov S.A. 2013.

Submitted June 15, 2013. 
domains with boundaries, DO and PDO far from being self-adjoint were studied in [3, 4, 1318]; degenerate elliptic problems were considered in [3, 4, 13].

2. In the present paper we study spectral properties of a non-self-adjoint operator in $L_{2}(0,1)^{l}$ associated with the sesquilinear form

$$
\mathcal{A}[u, v]=\sum_{i, j=0}^{m} \int_{0}^{1}<p_{i}(t) a_{i j}(t) u^{(i)}(t), p_{j}(t) v^{(j)}(t)>_{\mathbb{C}^{l}} d t .
$$

Here

$$
\begin{gathered}
p_{i}(t)=\{t(1-t)\}^{\theta+i-m} \quad(i=\overline{0, m}), \theta<m, u^{(i)}(t)=\frac{d^{i} u(t)}{d t^{i}}, \\
a_{i j} \in L_{\infty}\left(J ; \text { End } \mathbb{C}^{l}\right) \quad(i, j=\overline{0, m})
\end{gathered}
$$

where $J=(0,1)$. The symbol $<,>_{\mathbb{C}^{l}}$ denotes the scalar product in $\mathbb{C}^{l}$.

By $\mathcal{H}_{+}$we denote the closure of linear manifold $C_{0}^{\infty}(J)$ by the norm

$$
|\varphi|_{+}=\left(\int_{J} p_{m}^{2}(t)\left|\varphi^{(m)}(t)\right|^{2} d t+\int_{J}|\varphi(t)|^{2} d t\right)^{1 / 2} .
$$

We let

$$
\begin{gathered}
\mathcal{H}=L_{2}(J), \quad \mathcal{H}^{l}=\mathcal{H} \oplus \cdots \oplus \mathcal{H} \quad(\text { l times }), \\
\mathcal{H}_{+}^{l}=\mathcal{H}_{+} \oplus \cdots \oplus \mathcal{H}_{+} \quad(\text { l times }) .
\end{gathered}
$$

In what follows we denote the scalar product in the spaces $\mathcal{H}, \mathcal{H}^{l}$ by the same symbol (, ). In the same way, the norms in the spaces $\mathcal{H}_{+}, \mathcal{H}_{+}^{l}$ and $\mathcal{H}, \mathcal{H}^{l}, \mathbb{C}^{l}$ will be denoted respectively by ||$_{+},||$. By the symbol $\|T\|$ we shall denote the norm of a bounded operator $T$ defined either in $\mathcal{H}$ or in $\mathcal{H}^{l}$.

As the domain of sesquilinear form $\mathcal{A}[u, v](1.2)$ we take space $\mathcal{H}_{+}^{l}$.

Suppose that $a_{m m}(t) \in C^{m}\left(\bar{J} ; E n d \mathbb{C}^{l}\right)$ and for each $t \in \bar{J}$ the matrix $a(t)=a_{m m}(t)$ has $l$ different nonzero eigenvalues $\mu_{1}(t), \ldots, \mu_{l}(t)$. Then the eigenvalues of matrix $a(t)$ can be ordered so that $\mu_{j}(t), \mu_{j}^{-1}(t) \in C^{m}(\bar{J}), j=\overline{1, l}$.

Suppose the conditions

$$
\begin{gathered}
\left|a_{i j}(t)\right| \leqslant M t^{\delta}(1-t)^{\delta} \quad(i+j<2 m), \quad \delta>0, \\
\mu_{j}(t) \notin S \quad(j=\overline{1, l}, t \in \bar{J}),
\end{gathered}
$$

where $S \subset \mathbb{C}$ is a closed angle with the vertex at the origin and $\mu_{j}(t)$ are the eigenvalues of matrix $a(t)$.

Under the above conditions the following theorems hold true (see [1]).

Theorem 1.1. There exists the unique closed operator $A$ in $\mathcal{H}^{l}$ with the properties

(i) $\quad D(A) \subset \mathcal{H}_{+}^{l},(A u, v)=\mathcal{A}[u, v] \quad \forall u \in D(A), v \in \mathcal{H}_{+}^{l}$,

(ii) for some $z_{0} \in \mathbb{C}$ there exists the bounded inverse

$$
\left(A-z_{0} E\right)^{-1}: \mathcal{H}^{l} \rightarrow \mathcal{H}^{l} .
$$

Let $A$ be the operator from the previous theorem.

Theorem 1.2. Operator $A$ has a discrete spectrum. The system of root vector-functions of operator $A$ is complete in $\mathcal{H}^{l}$, i.e., their finite linear combinations are complete in $\mathcal{H}^{l}$. The order of the resolvent of operator $A$ does not exceed $\frac{1}{2 m}$. The number $N(\lambda)$ of the eigenvalues of operator $A$ whose moduli does not exceed $\lambda$ taken counting multiplicity satisfies the estimate $N(\lambda) \leqslant M \lambda^{1 / 2 m}, \lambda \geq 1$. 
We observe that in the case of a symmetric form (1.2) the above formulated results are well-known.

3. We denote by $\mathcal{H}_{-}$the completion of the space $\mathcal{H}$ by the norm

$$
|u|_{-}=\sup _{0 \neq \varphi \in \mathcal{H}_{+}} \frac{|(u, \varphi)|}{|\varphi|_{+}} .
$$

We let $\mathcal{H}_{-}^{l}=\mathcal{H}_{-} \oplus \cdots \oplus \mathcal{H}_{-}(l$ times $)$. An element $F=\left(F_{1}, \ldots, F_{l}\right) \in \mathcal{H}_{-}^{l}$ generates an antilinear functional over $\mathcal{H}_{+}^{l}$ by the formula

$$
<F, v>=\lim _{i \rightarrow+\infty}\left(u_{i}, v\right), \quad v \in \mathcal{H}_{+}^{l},
$$

where the sequence of vector functions $u_{1}, u_{2}, \ldots \in \mathcal{H}^{l}$ is chosen so that $u_{i} \rightarrow F(i \rightarrow+\infty)$ in $\mathcal{H}_{-}^{l}$.

We note that if $v=\left(v_{1}, \ldots, v_{l}\right) \in \mathcal{H}_{+}^{l}$, then

$$
<F, v>=\sum_{i=1}^{l}<F_{i}, v_{i}>, \quad|F|_{-}=\left(\sum_{i=1}^{l}\left|F_{i}\right|_{-}^{2}\right)^{1 / 2} .
$$

Hereinafter, both for $l=1$ and for an arbitrary $l \in N$ we adopt the same notations ||$_{-},<,>$.

And vice versa, for each continuous antilinear functional $g(v)\left(v \in \mathcal{H}_{+}^{l}\right)$ there exists the unique element $F \in \mathcal{H}_{-}^{l}$ such that $g(v)=\left\langle F, v>, \forall v \in \mathcal{H}_{+}^{l}\right.$. At that, the norm of functional $g$ is equal to $|F|_{-}$.

In what follows continuous antilinear functionals over $\mathcal{H}_{+}^{l}$ are identified with the elements of the space $\mathcal{H}_{-}^{l}$.

4. Under condition (1.3), by Hardy inequality we have

$$
|\mathcal{A}[u, v]| \leqslant M|u|_{+}|v|_{+} \quad\left(\forall u, v \in \mathcal{H}_{+}^{l}\right) .
$$

This is why we can introduce the operator $\mathcal{A}: \mathcal{H}_{+}^{l} \rightarrow \mathcal{H}_{-}^{l}$ acting by the formula

$$
<\mathcal{A} u, v>=\mathcal{A}[u, v] \quad\left(\forall u, v \in \mathcal{H}_{+}^{l}\right) .
$$

Let $A$ be the operator from Theorems 1.1, 1.2. The following theorem holds true.

Theorem 1.3. For $\lambda \in S$ with sufficiently large modulus there exists bounded inverses

$$
(\mathcal{A}-\lambda E)^{-1}: \mathcal{H}_{-}^{l} \rightarrow \mathcal{H}_{-}^{l}, \quad(A-\lambda E)^{-1}: \mathcal{H}^{l} \rightarrow \mathcal{H}^{l},
$$

and the identity

$$
(\mathcal{A}-\lambda E)^{-1} u=(A-\lambda E)^{-1} u \quad\left(\forall u \in \mathcal{H}^{l}\right)
$$

holds true. At that, $A u=\mathcal{A} u(\forall u \in D(A))$ and

$$
D(A)=\left\{u \in \mathcal{H}_{+}^{l}: \mathcal{A} u \in \mathcal{H}^{l}\right\} .
$$

Similar result for a scalar partial differential operator was obtained in work [19]. We note that that first part of Theorem 1.3 can be proven by the approach of paper [19] only in the case if one assumes additionally that for some continuous on $\bar{J}$ non-zero function $\gamma(t)$ and for sufficiently small $\varepsilon>0$ the condition

$$
\left|\arg \left\{\gamma(t)<a(t) h, h>_{\mathbb{C}^{l}}\right\}\right|<\frac{\pi-\varepsilon}{2} \quad\left(\forall t \in \bar{J}, 0 \neq h \in \mathbb{C}^{l}\right)
$$

holds true. Hereinafter we assume the function $\arg z$ takes the values in the segment $(-\pi, \pi]$.

In particular, it follows from (1.4) that

$$
\left|\arg \gamma(t) \mu_{j}(t)\right| \leqslant \frac{\pi-\varepsilon}{2} \quad(\forall t \in \bar{J}, j=\overline{1, l}) .
$$

5. The proof of Theorem 1.2 is given in Section 2. We note that in Section 2 we also prove the following estimate for the resolvent of operator $A$ in sector $S$ :

$$
\left\|(A-\lambda E)^{-1}\right\| \leqslant M|\lambda|^{-1}, \quad(\lambda \in S,|\lambda| \geq c(S)),
$$


where $c(S)>0$. The summability of Fourier series for the elements $f \in \mathcal{H}^{l}$ in terms of root vector functions of operator $A$ by the Abel methods with brackets was established in [1]. In the present work we prove the completeness for the system of root vector functions of operator $A$ in space $\mathcal{H}^{l}$.

In Section 3 we describe the domain of operator $A$. In Section 4 we study the asymptotic behavior of the eigenvalues of operator $A$.

The results of this work were announced partially in [20].

\section{Estimate For Resolvent of operator $A$}

1. Let $P$ be the self-adjoint operator in $\mathcal{H}$ associated with the sesquilinear form

$$
P^{\prime}[u, v]=\left(\rho^{\theta} u^{(m)}, \rho^{\theta} v^{(m)}\right), D\left[P^{\prime}\right]=\mathcal{H}_{+},
$$

where $\rho(t)=t(1-t), t \in[0,1], \theta$ is the same as in (1.2).

We denote (see [1]) by $\mathcal{H}_{\nu}^{r}, \nu>0$, the space of functions $u \in \mathcal{H}_{+}^{r}$ with norm

$$
|u|_{\nu}=\left(\int_{J} \rho^{2 \theta}(t)\left|u^{(m)}(t)\right|^{2} d t+\nu \int_{J}|u(t)|^{2} d t\right)^{1 / 2} .
$$

We indicate by $\mathcal{H}_{-\nu}^{r}, \nu>0$, the space of elements $F \in \mathcal{H}_{-}^{r}$ with the norm

$$
|F|_{-\nu, r}=\sup _{\substack{v \in \mathcal{H}_{+}^{r} \\|v|_{\nu} \leqslant 1}}|\langle F, v\rangle| .
$$

As $\nu_{1}, \nu_{2}>0$, the sets $\mathcal{H}_{ \pm \nu_{1}}^{r}, \mathcal{H}_{ \pm \nu_{2}}^{r}$ coincide, while as the normed spaces they differ only by the equivalent norms. For $\nu=1$ we have $\mathcal{H}_{\nu}^{r}=\mathcal{H}_{+}^{r}, \mathcal{H}_{-\nu}^{r}=\mathcal{H}_{-}^{r}$. Space $\mathcal{H}_{-\nu}^{r}, \nu>0$, is the negative one in the triple $\mathcal{H}_{\nu}^{r} \subset \mathcal{H}^{r} \subset \mathcal{H}_{-\nu}^{r}$ w.r.t. the positive space $\mathcal{H}_{\nu}^{r}$ (see, for instance, [21]).

In what follows we shall make use of the following lemma (see [1]).

Lemma 2.1. There exists the bounded inverse operator $T_{\omega}: \mathcal{H}_{-} \rightarrow \mathcal{H}, \omega \geq 1$, such that $T_{\omega} u=(P+\omega E)^{-\frac{1}{2}} u, \forall u \in \mathcal{H}$, at that

$$
\left|T_{\omega} F\right| \leqslant M|F|_{-\nu}, \quad \forall \omega \geq 1, \nu \in[1,2 \omega), \quad \forall F \in \mathcal{H}_{-\nu},
$$

where the number $M>0$ is independent of $\omega, \nu$.

2. Let $T_{\omega}$ be the operator from $2.1, \mathcal{T}_{\omega}: \mathcal{H} \rightarrow \mathcal{H}_{-}$be the inverse operator for $T_{\omega}: \mathcal{H}_{-} \rightarrow \mathcal{H}$. As in Lemma 2.1, one can prove that

$$
\left.\left|\mathcal{T}_{\omega} u\right|_{-\nu} \leqslant M|u| \quad \forall \omega \geq 1, \quad \nu \in[1,2 \omega), \quad \forall u \in \mathcal{H}\right),
$$

where number $M>0$ is independent of $\omega, \nu$. At that, if $u \in \mathcal{H}_{\nu}$, then

$$
\mathcal{T}_{\omega} u=(P+\omega E)^{\frac{1}{2}} u .
$$

We introduce the operators $T_{\omega}^{l}: \mathcal{H}_{-}^{l} \rightarrow \mathcal{H}^{l}, \mathcal{T}_{\omega}^{l}: \mathcal{H}^{l} \rightarrow \mathcal{H}_{-}^{l}$ by the formulae

$$
T_{\omega}^{l}=\operatorname{diag}\left\{T_{\omega}, \ldots, T_{\omega}\right\}, \quad \mathcal{T}_{\omega}^{l}=\operatorname{diag}\left\{\mathcal{T}_{\omega}, \ldots, \mathcal{T}_{\omega}\right\}
$$

We let $P_{l}=\operatorname{diag}\{P, \ldots, P\}$.

Theorem 2.1. For $\lambda \in S,|\lambda| \geq \sigma$, where $\sigma>0$ is a sufficiently large number the representations

$$
\begin{gathered}
(\mathcal{A}-\lambda E)^{-1}=\left(P_{l}+|\lambda| E\right)^{-1} \Phi(\lambda) T_{\lambda} \\
(A-\lambda E)^{-1}=\left(P_{l}+|\lambda| E\right)^{-\frac{1}{2}} \Phi(\lambda)\left(P_{l}+|\lambda| E\right)^{-\frac{1}{2}},
\end{gathered}
$$

hold true, where $\Phi(\lambda): \mathcal{H}^{l} \rightarrow \mathcal{H}^{l}$ is a bounded operator

$$
\sup _{\lambda \in S,|\lambda| \geq \sigma}\|\Phi(\lambda)\|<+\infty
$$


Proof. Let us prove that if $\nu=|\lambda|$, then for $\lambda \in S$ with sufficiently large modulus we have

$$
\left|\left(P_{l}+|\lambda| E\right)^{\frac{1}{2}}\left(\mathcal{A}_{\nu}-\lambda E\right)^{-1} \mathcal{T}_{|\lambda|}^{l} u\right| \leqslant M|u| \quad \forall u \in \mathcal{H}^{l} .
$$

In order to it, we employ the identity (see [1, Sec. 4, Eqs. (4.6), (4.7)])

$$
\left(\mathcal{A}_{\nu}-\lambda E\right)^{-1}=X_{\nu}(\lambda) \Gamma_{\nu}^{\prime}(\lambda) .
$$

Here the operator $\mathcal{A}_{\nu}: \mathcal{H}_{\nu}^{l} \rightarrow \mathcal{H}_{-\nu}^{l}$ is defined by the formula

$$
<\mathcal{A}_{\nu} u, v>=\mathcal{A}[u, v], \quad\left(\forall u, v \in \mathcal{H}_{\nu}^{l}\right) .
$$

It is clear that

$$
\left|T_{|\lambda|}^{l} \Gamma_{|\lambda|}^{(\lambda)} \mathcal{T}_{|\lambda|}^{l} u\right| \leqslant\left|\Gamma_{|\lambda|}^{(\lambda)} \mathcal{T}_{|\lambda|}^{l} u\right|_{-|\lambda|} \leqslant M_{1}\left|\mathcal{T}_{|\lambda|}^{l} u\right|_{-|\lambda|} \leqslant M_{2}|u|, \quad \lambda \in S,|\lambda| \geq \sigma_{1} .
$$

It remains to show (see $[1$, Sec. 4 , Eqs. (4.6), (4.7)]) that

$$
\left|\left(P_{l}+|\lambda| E\right)^{\frac{1}{2}} X_{|\lambda|}(\lambda) \mathcal{T}_{|\lambda|}^{l} u\right| \leqslant M_{3}|u|, \quad u \in \mathcal{H}^{l} .
$$

Employing (4.3), (3.13) from [1], as above, we reduce the proof of estimate (2.3) to checking the following inequality

$$
\left|\left(P_{l}+|\lambda| E\right)^{\frac{1}{2}} R_{k,|\lambda|}(\lambda) \mathcal{T}_{|\lambda|} v\right| \leqslant M_{4}|v|, \quad v \in \mathcal{H} .
$$

The validity of this inequality for $\lambda \in S$ with sufficiently large modulus follows from representation (3.12) in work [1]. Thus, we have

$$
\left(\mathcal{A}_{\nu}-\lambda E\right)^{-1}=\left(P_{l}+|\lambda| E\right)^{-\frac{1}{2}} \Phi(\lambda) T_{|\lambda|}^{l}, \quad \lambda \in S,|\lambda| \geq \sigma,
$$

where $\Phi(\lambda): \mathcal{H}^{l} \rightarrow \mathcal{H}^{l}$ is a bounded operator obeying estimate (2.2).

We note that

$$
\left(\mathcal{A}_{\nu}-\lambda E\right)^{-1} F=(\mathcal{A}-\lambda E)^{-1} F . \quad \forall \nu \geq 1, F \in \mathcal{H}_{-}^{l} .
$$

For $u \in \mathcal{H}^{l}$ we get

$$
T_{|\lambda|}^{l} u=\left(P_{l}+|\lambda| E\right)^{-\frac{1}{2}} u, \quad(\mathcal{A}-\lambda E)^{-1} u=(A-\lambda E)^{-1} u
$$

and together with $(2.4),(2.5)$ it proves $(2.1),\left(2.1^{\prime}\right)$. The proof is complete.

3. Representation $\left(2.1^{\prime}\right)$ implies the estimate

$$
\left\|(A-\lambda E)^{-1}\right\| \leqslant M|\lambda|^{-1} . \quad \lambda \in S,|\lambda| \geq \sigma .
$$

Since the order of the resolvent of operator $P_{l}$ equals $\frac{1}{2 m}$, it follows from $\left(2.1^{\prime}\right)$ that the order of the resolvent of operator $A$ does not exceed the number $\frac{1}{2 m}$. Applying Theorem 6.4.1 in [10], by (2.6) we conclude that the system of root vector functions of operator $A$ is complete in $\mathcal{H}^{l}$.

We note the summability of Fourier series by Abel method with brackets w.r.t. the system of root vector functions of operator $A$ was established in [1].

4. Let $H$ be a separable Hilbert space. We denote by $\sigma_{\tau}(H), \tau \geq 1$, the class of operators $L \in \sigma_{\infty}(H)$ for $s$-numbers are summable with the power $\tau[22]$ :

$$
\|L\|_{\tau}=\left(\sum_{j=1}^{\infty} s_{j}^{\tau}(L)\right)^{\frac{1}{\tau}}<+\infty .
$$

The infimum of the numbers $\tau$ such that $L \in \sigma_{\tau}(H)$ is called the order of operator $L$.

We indicate by $\nu_{1}(t), \nu_{2}(t), \ldots$ the eigenvalues of operator $L \in \sigma_{\infty}(H)$ taken in the order of ascending modulus counting their root multiplicities. We note that

$$
\nu_{j}\left(\left(L^{*} L\right)^{\frac{1}{2}}\right)=s_{j}(L), \quad j=1,2, \ldots
$$


In what follows we shall make use of well-known inequalities (see, for instance, [22]):

$$
\begin{gathered}
\sum_{j=1}^{+\infty}\left|\mu_{j}(t)\right| \leqslant\|L\|_{1}, \quad \forall L \in \sigma_{1}(H), \\
\left\|L L^{\prime}\right\|_{p} \leqslant\|L\|_{p}\left\|L^{\prime}\right\|,\left\|L^{\prime} L\right\|_{p} \leqslant\left\|L^{\prime}\right\|\|L\|_{p},
\end{gathered}
$$

if $L \in \sigma_{p}(H), p \geq 1, L^{\prime}$ is a bounded operator;

$$
\left\|L_{1} \ldots L_{r}\right\|_{p} \leqslant\left\|L_{1}\right\|_{\kappa_{1}} \ldots\left\|L_{r}\right\|_{\kappa_{r}}
$$

if $L_{j} \in \sigma_{\kappa_{j}}(H), 1 \leqslant p \leqslant \kappa_{j}, j=\overline{1, r}, \sum_{j=1}^{r} \kappa_{j}^{-1}=\frac{1}{p}$. As $L_{1}=\ldots=L_{r}=L \in \sigma_{1}(H), \kappa_{j}=r$, $j=\overline{1, r})$, thanks to $(2.9)$ we obtain inequality

$$
\left\|L^{r}\right\|_{1} \leqslant\|L\|_{r}^{r}
$$

By (2.7) we obtain the convergence of the series

$$
\operatorname{sp} L \stackrel{\text { def }}{=} \sum_{j=1}^{+\infty} \nu_{j}(t), \quad \forall L \in \sigma_{1}(H)
$$

5. In conclusion of this section let us prove the statement of Theorem 1.2 on the estimate for the spectrum of operator $A$.

We denote by $\lambda_{1}, \lambda_{2}, \ldots$ the eigenvalues of the operator $A$ taken in order of ascending moduli counting the root multiplicity.

Employing $\left(2.1^{\prime}\right),(2.8)-(2.10)$, we find

$$
\begin{gathered}
\left\|(A-\lambda E)^{-r}\right\|_{1} \leqslant\left\|\left(P_{l}+|\lambda| E\right)^{-\frac{1}{2}} \Phi(\lambda)\left(P_{l}+|\lambda| E\right)^{-\frac{1}{2}}\right\|_{r}^{r} \leqslant M\left\|\left(P_{l}+|\lambda| E\right)^{-\frac{1}{2}}\right\|_{2 r}^{2 r}, \\
\lambda \in S,|\lambda| \geq \sigma,
\end{gathered}
$$

where $r=4 m, \sigma>0$ is a sufficiently large number. It is known that

$$
N_{0}(t) \stackrel{\text { def }}{=} \sum_{\omega_{j} \leqslant t} 1 \sim \text { const } \cdot t^{\frac{1}{2 m}}(t \rightarrow+\infty),
$$

where $\omega_{1}, \omega_{2}, \ldots$ denotes the eigenvalues of operator $P$. This is why

$$
\left\|\left(P_{l}+|\lambda| E\right)^{-\frac{1}{2}}\right\|_{8 m}^{8 m}=\sum_{j=1}^{+\infty}\left(\omega_{j}+|\lambda|\right)^{-4 m}=\int_{0}^{+\infty} \frac{d N_{0}(t)}{(t+|\lambda|)^{4 m}} \leqslant M|\lambda|^{\frac{1}{2 m}-4 m} \quad|\lambda| \geq 1 .
$$

By (2.7), (2.11) it implies that

$$
\sum_{j=1}^{+\infty}\left|\left(\lambda_{j}-\lambda\right)^{-4 m}\right| \leqslant M|\lambda|^{\frac{1}{2 m}-4 m} \quad \lambda \in S, \quad|\lambda| \geq \sigma
$$

We choose a number $\varphi \in(-\pi ; \pi]$ so that the ray $\Gamma=\left\{\lambda=t e^{i \varphi}: t \geq 0\right\}$ is the bisectrix of angle $S$. Then

$$
|z|+|\lambda| \leqslant c^{\prime}|z-\lambda| \quad \forall z \notin S, \quad \lambda \in \Gamma,
$$

where number $c^{\prime}>0$ depends only on angle $S$. For sufficiently large $j \geq j_{0}$ we have $\lambda_{j} \notin S$. It is obvious that

$$
\begin{aligned}
N(t)=\int_{0}^{t} d N(\tau) \leqslant & (2 t)^{4 m} \int_{0}^{t} \frac{d N(\tau)}{(t+\tau)^{4 m}} \leqslant(2 t)^{4 m} \int_{0}^{+\infty} \frac{d N(\tau)}{(t+\tau)^{4 m}}= \\
& =(2 t)^{4 m} \sum_{j=1}^{+\infty}\left(\left|\lambda_{j}\right|+t\right)^{-4 m},
\end{aligned}
$$


where $N(t)=\operatorname{card}\left\{j:\left|\lambda_{j}\right| \leqslant t\right\}$. Therefore, (see (2.12))

$$
N(t) \leqslant M_{1}+M_{2} t^{4 m} \sum_{j=j_{0}}^{+\infty}\left|\lambda_{j}-t e^{i \varphi}\right|^{-4 m} \leqslant M_{2} t^{\frac{1}{2 m}}(t \geq 1) .
$$

The proof of Theorem 1.2 is complete.

\section{DESCRIPTION OF DOMAin OF Operator $A$}

1. Let $A$ be the operator from Theorem 1.1 and

$$
a_{i j}(t) \in C^{j}\left(J ; E n d \mathbb{C}^{l}\right) \quad i, j=\overline{0, m} .
$$

Theorem 3.1. The domain $D(A)$ of operator $A$ is the class of vector functions $u \in$ $W_{2, l o c}^{2 m}(J)^{l} \cap \mathcal{H}_{+}^{l}$ such that

$$
f=\sum_{i, j=0}^{m}(-1)^{j}\left(p_{i}(t) p_{j}(t) a_{i j}(t) u^{(i)}(t)\right)^{(j)} \in \mathcal{H}^{l} .
$$

At that, $f=A u$.

Proof. Let $u \in W_{2, l o c}^{2 m}(J)^{l} \cap \mathcal{H}_{+}^{l}$ and $f(t) \in \mathcal{H}^{l}$. Then integrating by parts for an arbitrary vector function $v(t) \in C_{0}^{\infty}(J)^{l}$, we obtain

$$
(f, v)=\sum_{i, j=0}^{m}\left(p_{i}(t) a_{i j}(t) u^{(i)}(t), p_{j}(t) v^{(j)}(t)\right)=\mathcal{A}[u, v] .
$$

By continuity these identities are valid for each $v \in \mathcal{H}_{+}^{l}$. Hence, in accordance with Theorem 1.1, $u \in D(A), f=A u$.

And vice versa, let $u \in D(A), f_{1}=A u$. Then

$$
\left(f_{1}, v\right)=\sum_{i, j=0}^{m}\left(p_{i} a_{i j} u^{(i)}, p_{j} v^{(j)}\right), \quad \forall v \in C_{0}^{\infty}(J)^{l},
$$

so the element

$$
f_{2}=\sum_{i, j=0}^{m}(-1)^{j}\left(p_{i}(t) p_{j}(t) a_{i j}(t) u^{(j)}(t)\right)^{(j)}
$$

treated in the distribution sense belongs to $\mathcal{H}^{l}$. At that we have $f_{1}=f_{2}$. Then by the general theory of elliptic equations we get $u \in W_{2, l o c}^{2 m}(J)^{l}$.

2. In relation with Theorem 3.1 we note that as $-\frac{1}{2}<\theta<m-\frac{1}{2}$, space $\mathcal{H}_{+}^{l}$ is described (see [23]) as the class of vector functions $u(t) \in \mathcal{H}^{l}$ with the finite norm

$$
|u|_{+}=\left(\int_{J}\left|\rho^{2 \theta}(t) u(t)\right|^{2} d t+\int_{J}|u(t)|^{2} d t\right)^{\frac{1}{2}}<+\infty
$$

and having zero traces

$$
u^{(j)}(0)=u^{(j)}(1)=0, \quad j=0,1, \ldots, s_{0}-1 ;
$$

here $s_{0}$ is an integer such that $m-\theta-\frac{1}{2} \leqslant s_{0}<m-\theta+\frac{1}{2}$. If $\theta \leqslant-\frac{1}{2}$ or $m-\frac{1}{2} \leqslant \theta<m$, then space $\mathcal{H}_{+}^{l}$ comprises vector functions $u(t) \in \mathcal{H}^{l}$ (see [23]) with finite norm $|u|_{+}$.

3. Together with Theorem 3.1 we also have 
Theorem 3.2. Suppose (3.1) and

$$
\left|a_{i j}^{(k)}(t)\right| \leqslant M\{t(1-t)\}^{-k},(k=0,1, \ldots, j) .
$$

Moreover, let $\theta+\frac{1}{2} \notin\{1,2, \ldots, m\}$. Then the domain of operator $A$ is described as the class of vector functions $u \in W_{2, l o c}^{2 m}(J)^{l} \cap \mathcal{H}_{+}^{l}$ such that

$$
p_{0}(t) u(t), \quad \sum_{i, j=0}^{m}(-1)^{j}\left(p_{i}(t) p_{j}(t) a_{i j}(t) u^{(j)}(t)\right)^{(j)} \in \mathcal{H}^{l} .
$$

\section{Asymptotic distribution of eigenvalues of operator $A$}

1. Let $A$ be the operator from Theorem 1.1. Suppose that the eigenvalues $\mu_{1}(t), \ldots, \mu_{l}(t)$ of matrix $a(t)$ are located in the complex plane as follows

$$
\mu_{1}(t), \ldots, \mu_{n}(t) \in R_{+} \stackrel{\text { def }}{=}\{z \in \mathbb{C}: \operatorname{Re}, z>0, \operatorname{Im} z=0\}, \quad \mu_{n+1}(t), \ldots, \mu_{l}(t) \notin \bar{\Phi},
$$

where $1 \leqslant n \leqslant l, \Phi=\{z \in \mathbb{C}:|\arg z|<\varphi\}, \varphi \in(0, \pi)$. Then in accordance with Theorem 1.3 each closed sector $S \subset \bar{\Phi} \backslash R_{+}$with the vertex at the origin contains a finite number of eigenvalues of operator $A$. It implies easily that

$$
\lim _{j \rightarrow+\infty} \arg \lambda_{j}=0
$$

where $\lambda_{1}, \lambda_{2}, \ldots$ are the eigenvalues of operator $A$ in angle $\Phi$ taken in the order of ascending moduli counting root multiplicity.

Theorem 4.1. Ast $\rightarrow+\infty$, function

$$
N(t)=\operatorname{card}\left\{j:\left|\lambda_{j}\right| \leqslant t\right\}
$$

satisfies asymptotic formula

$$
N(t) \sim c t^{\frac{1}{2 m}}, \quad c=\frac{1}{\pi} \sum_{j=1}^{n} \int_{0}^{1} \rho^{-\frac{\theta}{m}}(t) \mu_{j}^{-\frac{1}{2 m}}(t) d t .
$$

Similar result for second order differential operator was obtained in $[4,13]$. We however note that the approach of works $[4,13]$ can not be directly employed in the case $m>1$ even if condition (1.4) holds true. A key point of our approach is that we "extract" explicitly the main term of the "generalized" resolvent as an operator acting from $\mathcal{H}_{-\nu}^{l}$ into $\mathcal{H}_{\nu}^{l}$.

We note also that similar result for a class of non-self-adjoint elliptic systems was proven in [24].

In combination with some other analytic approaches, it allows us to calculate the main term of the asymptotics for the function $\operatorname{sp}(A-z E)^{-1}$ as $z \rightarrow+\infty$ along some rays $\Gamma \subset \bar{\Phi} \backslash R_{+}$ starting at the origin. The asymptotic formulae established in this way relate to classes of operators wider than in works $[4,13]$ even in the case $m=1$.

2. To prove Theorem 4.1 we employ (4.6), (4.7) from [1] as $\nu=|\lambda|$. Let $P_{l}, T_{\omega}^{l}, \mathcal{T}_{\omega}^{l}$ be the same operators like in Item 1 of Section 2.

We denote by $u_{1}, u_{2}, \ldots$ the orthonormalized vector eigenfunctions of operator $P_{l}$. Let $P_{l} u_{j}=$ $\omega_{j} u_{j}, \omega_{1} \leqslant \omega_{2} \leqslant \ldots$ Since $u_{1}, u_{2}, \ldots$ is an orthonormalized basis in $\mathcal{H}^{l}$ and $(A-\lambda E)^{-1} u_{j}=$ $\left(\mathcal{A}_{\nu}-\lambda E\right)^{-1} u_{j} \quad \forall \nu \geq 1$, then

$$
\begin{aligned}
& \operatorname{sp}(A-\lambda E)^{-1}=\sum_{j=1}^{+\infty}\left((A-\lambda E)^{-1} u_{j}, u_{j}\right)=\sum_{j=1}^{+\infty}\left(\left(\mathcal{A}_{\nu}-\lambda E\right)^{-1} u_{j}, u_{j}\right)= \\
= & \sum_{j=1}^{+\infty}\left(X_{\nu}(\lambda) u_{j}, u_{j}\right)+\sum_{j=1}^{+\infty}\left(X_{\nu}(\lambda) \Gamma_{\nu}(\lambda) u_{j}, u_{j}\right), \quad \lambda \in S, \quad|\lambda| \geq \sigma=\sigma(S),
\end{aligned}
$$


where $S \subset \bar{\Phi} \backslash R_{+}$is an arbitrary closed angle with the vertex at the origin. Taking into consideration

$$
\left(P_{l}+|\lambda| E\right)^{ \pm \frac{1}{2}} u_{j}=\left(\omega_{j}+|\lambda|\right)^{ \pm \frac{1}{2}} u_{j}
$$

we obtain

$$
\begin{aligned}
\sum_{j=1}^{+\infty}\left(X_{\nu}(\lambda) \Gamma_{\nu}(\lambda) u_{j}, u_{j}\right) & =\sum_{j=1}^{+\infty}\left(X_{\nu}(\lambda) \Gamma_{\nu}(\lambda)\left(P_{l}+|\lambda| E\right)^{\frac{1}{2}} u_{j},\left(P_{l}+|\lambda| E\right)^{-\frac{1}{2}} u_{j}\right) \\
& =\sum_{j=1}^{+\infty}\left(\left(P_{l}+|\lambda| E\right)^{-\frac{1}{2}} X_{\nu}(\lambda) \mathcal{T}_{|\lambda|} T_{|\lambda|} \Gamma_{\nu}(\lambda) \mathcal{T}_{|\lambda|} u_{j}, u_{j}\right)
\end{aligned}
$$

According to (4.6) (see [1, Sec. 4]), as $\nu=|\lambda|, u \in \mathcal{H}^{l}$ we have

$$
\left|\mathcal{T}_{|\lambda|} \Gamma_{\nu}(\lambda) T_{|\lambda|} u\right| \leqslant M\left|\Gamma_{\nu}(\lambda) T_{|\lambda|} u\right|_{-|\lambda|} \leqslant M_{1}|\lambda|^{-\varepsilon^{\prime}}\left|T_{|\lambda|} u\right|_{-|\lambda|}=M_{2}|\lambda|^{-\varepsilon^{\prime}}|u| .
$$

Hence, the operator $\mathcal{T}_{|\lambda|} \Gamma_{\nu}(\lambda) T_{|\lambda|}$ induces a bounded operator in $\mathcal{H}^{l}$ with the norm not exceeding $M_{2}|\lambda|^{-\varepsilon^{\prime}}$. In view of (4.1), (4.2), we thus find

$$
Z(\lambda) \stackrel{\text { def }}{=}\left|\operatorname{sp}(A-\lambda E)^{-1}-\sum_{j=1}^{+\infty}\left(X_{\nu}(\lambda) u_{j}, u_{j}\right)\right| \leqslant M|\lambda|^{-\varepsilon^{\prime}}\left\|\left(P_{l}+|\lambda| E\right)^{-\frac{1}{2}} X_{\nu}(\lambda) \mathcal{T}_{|\lambda|}\right\|_{1} .
$$

Although here $\mathcal{T}_{|\lambda|}$ is an unbounded operator in $\mathcal{H}^{l}$, the operator $X_{\nu}(\lambda) \mathcal{T}_{|\lambda|}$ induces a bounded operator in $\mathcal{H}^{l}$. Applying (2.3), we find

$$
Z(\lambda) \leqslant M|\lambda|^{-\varepsilon^{\prime}}\left|\left(P_{l}+|\lambda| E\right)^{-1}\right|_{1} \leqslant M_{1}|\lambda|^{\frac{1}{2 m}-1-\varepsilon^{\prime}} .
$$

We then have (see [1, Sec. 4, Eq. (4.3)])

$$
\begin{aligned}
\sum_{j=1}^{+\infty}\left(X_{\nu}(\lambda) u_{j}, u_{j}\right) & =\sum_{j=1}^{+\infty}\left(U\left(\mathcal{B}_{\nu}-\lambda E\right)^{-1} U^{-1} u_{j}, u_{j}\right) \\
& =\sum_{j=1}^{+\infty}\left(\left(\mathcal{B}_{\nu}-\lambda E\right)^{-1} u_{j}, u_{j}\right)=\sum_{k=1}^{l} s p\left(\widetilde{Q}_{k}-\lambda E\right)^{-1} .
\end{aligned}
$$

Here the operators $\widetilde{Q}_{k}, k=\overline{1, l}$, are defined in space $\mathcal{H}$ as follows

$$
D\left(\widetilde{Q}_{k}\right)=\left\{v \in \mathcal{H}_{+}: Q_{\nu, k} v \in \mathcal{H}\right\}, \quad \forall \nu \geq 1, \quad \widetilde{Q}_{k} v=Q_{\nu, k} v, \quad \forall v \in D\left(\widetilde{Q}_{k}\right) .
$$

Operators $Q_{\nu, k}$ were introduced in [1, Sec. 4, Subsec. 1]. We remind that

$$
\left(\mathcal{B}_{\nu}-\lambda E\right)^{-1}=\operatorname{diag}\left\{\left(Q_{\nu, 1}-\lambda E\right)^{-1}, \ldots,\left(Q_{\nu, l}-\lambda E\right)^{-1}\right\} .
$$

The above introduced operators $\widetilde{Q}_{1}, \ldots, \widetilde{Q}_{l}$ are independent of number $\nu \geq 1$.

We employ Theorem 1.3 for the situation when $l=1, A=\widetilde{Q}_{j}, j=\overline{1, l}$. Then we obtain that in angle $\Phi$ operator $\widetilde{Q}_{j}, j=\overline{n+1, l}$ has a finite number of the eigenvalues. Since $\mu_{j}(t) \in$ $R_{+}(j=\overline{1, n})$, then $\widetilde{Q}_{j}=\widetilde{Q}_{j}^{*} \geq 0, j=\overline{1, n}$. Thus, we have

$$
\operatorname{sp}(A-\lambda E)^{-1}=\sum_{i=1}^{+\infty} \sum_{k=1}^{l}\left(\lambda_{i, k}-\lambda\right)^{-1}+O\left(|\lambda|^{\frac{1}{2 m}-1-\varepsilon^{\prime}}\right), \quad \lambda \in S, \quad|\lambda| \geq \sigma(S),
$$

where $\varepsilon^{\prime}>0, S \subset \bar{\Phi} \backslash R_{+}$is a closed angle with the vertex at the origin and $\lambda_{1, k}, \lambda_{2, k}, \ldots$ are the eigenvalues of operator $\widetilde{Q}_{k}$ taken in the order of ascending moduli.

Let $\psi \in(0, \varphi)$,

$$
\mathcal{L}=\{z \in \mathbb{C}: \arg z= \pm \psi\} \cup\{0\}
$$

is a contour enveloping $R_{+}$from the left. We choose numbers $c, \delta>0$ to satisfy the conditions 
(i) $\left|\left(\arg \lambda_{j}^{\prime}\right) \pm \varphi\right| \geq \delta,\left|\left(\arg \lambda_{j, k}\right) \pm \varphi\right| \geq \delta$, if respectively $\left|\lambda_{j}^{\prime}\right| \geq c$ or $\left|\lambda_{j, k}\right| \geq c, j=1,2, \ldots$, $k=\overline{1, l}$.

(ii) $\quad \lambda_{j, k} \notin \Phi,(k=\overline{n+1, l})$, if $\left|\lambda_{j, k}\right| \geq c$.

Here $\lambda_{1}^{\prime}, \lambda_{2}^{\prime}, \ldots$ are the eigenvalues of operator $A$ taken in the order of ascending moduli.

Then in the case $\left|\lambda_{j}^{\prime}\right| \geq c,\left|\lambda_{j, k}\right| \geq c$, for $\lambda \in \mathcal{L}$ we have $\left|\lambda-\lambda_{j}^{\prime}\right|^{-1} \leqslant M\left|\lambda_{j}^{\prime}\right|^{-\tau}|\lambda|^{\tau-1}$, $\left|\lambda-\lambda_{j, k}\right|^{-1} \leqslant M\left|\lambda_{j, k}\right|^{-\tau}|\lambda|^{\tau-1}$, where $\tau \in\left(\frac{1}{2 m}, 1\right)$. Hence,

$$
\begin{gathered}
\sum_{q \leqslant\left|\lambda_{j}^{\prime}\right|}\left|\lambda_{j}^{\prime}-\lambda\right|^{-1} \leqslant M_{1} r(q)|\lambda|^{\tau-1}, \\
r(q) \stackrel{\text { def }}{=} \sum_{q \leqslant\left|\lambda_{j}^{\prime}\right|}\left|\lambda_{j}^{\prime}\right|^{-\tau} \rightarrow 0, \quad q \rightarrow+\infty .
\end{gathered}
$$

Here we have employed the statement of Theorem 1.2 on the estimate for the spectrum of operator $A$.

We then have

$$
\frac{1}{2 \pi i} \int_{\mathcal{L}}\left(\sum_{a<\left|\lambda_{j}^{\prime}\right| \leqslant q}(t+\lambda)^{-1}\left(\lambda-\lambda_{j}^{\prime}\right)^{-1}\right) d \lambda=\sum_{a<\left|\lambda_{j}^{\prime}\right| \leqslant q}{ }^{\prime}\left(t+\lambda_{j}^{\prime}\right)^{-1},
$$

where the symbol $\sum^{\prime}$ denotes the summation over only $j$ satisfying $\left|\arg \lambda_{j}^{\prime}\right|<\psi$.

Taking into consideration that (see (4.4), (4.5))

$$
\lim _{q \rightarrow+\infty} r(q) \int_{\mathcal{L}}|\lambda|^{\tau-1}|t+\lambda|^{-1} d \lambda=0
$$

and passing in (4.6) to the limit as $q \rightarrow+\infty$, we find

$$
\frac{1}{2 \pi i} \int_{\mathcal{L}}(t+\lambda)^{-1}\left(\sum_{a<\left|\lambda_{j}^{\prime}\right|}\left(\lambda-\lambda_{j}^{\prime}\right)^{-1}\right) d \lambda=\sum_{a<\left|\lambda_{j}^{\prime}\right|}^{\prime}\left(t+\lambda_{j}^{\prime}\right)^{-1} .
$$

In the same way we get

$$
\frac{1}{2 \pi i} \int_{\mathcal{L}}(t+\lambda)^{-1}\left(\sum_{a<\left|\lambda_{j, k}\right|}\left(\lambda-\lambda_{j, k}\right)^{-1}\right) d \lambda=\sum_{a<\left|\lambda_{j, k}\right|}{ }^{\prime \prime}\left(t+\lambda_{j, k}\right)^{-1}, k=\overline{1, l},
$$

where the symbol $\sum^{\prime \prime}$ indicates the summation over such indices $j$ satisfying $\left|\arg \lambda_{j, k}\right| \leqslant \psi$.

Operators $\widetilde{Q}_{n+1}, \ldots, \widetilde{Q}_{l}$ have a finite number of eigenvalues in angle $\Phi$. In view of this fact and by $(4.3),(4.7),(4.8)$ we conclude that

$$
\sum_{j=1}^{+\infty}\left(t+\lambda_{j}\right)^{-1}=\sum_{j=1}^{+\infty} \sum_{k=0}^{n}\left(t+\lambda_{j, k}\right)^{-1}+O\left(t^{\frac{1}{2 m}-1-\varepsilon^{\prime}}\right), \quad t \rightarrow+\infty .
$$

Since $\arg \lambda_{j} \rightarrow 0(j \rightarrow+\infty)$, then $\lambda_{j}\left|\lambda_{j}\right|^{-1} \rightarrow 1, j \rightarrow+\infty$. Hence, for $q=1,2, \ldots$ we have

$$
\begin{aligned}
\sum_{j=q}^{+\infty}\left|\left(t+\lambda_{j}\right)^{-1}-\left(t+\left|\lambda_{j}\right|\right)^{-1}\right| & \leqslant 2 \sum_{j=q}^{+\infty}\left\{\frac{\left|\lambda_{j}-\right| \lambda_{j} \mid}{\left(t+\left|\lambda_{j}\right|\right)^{2}}\right\} \\
& \leqslant c_{q} \sum_{j=q}^{+\infty} \frac{\left|\lambda_{j}\right|}{\left(t+\left|\lambda_{j}\right|\right)^{2}} \leqslant c_{q}^{\prime} \sum_{j=q}^{+\infty}\left(t+\left|\lambda_{j}\right|\right)^{-1}
\end{aligned}
$$


where $c_{q}, c_{q}^{\prime} \rightarrow 0, q \rightarrow+\infty$. It easily implies that

$$
\sum_{j=1}^{+\infty}\left(t+\lambda_{j}\right)^{-1} \sim \sum_{j=1}^{+\infty}\left(t+\left|\lambda_{j}\right|\right)^{-1} \quad t \rightarrow+\infty .
$$

For the eigenvalues of operators $Q_{k}, k=\overline{1, n}$, the following estimate

$$
\sum_{j=1}^{+\infty}\left(t+\lambda_{j, k}\right)^{-1} \sim \int_{0}^{+\infty} \frac{d N_{j}(\tau)}{\tau+t}, \quad t \rightarrow+\infty,
$$

is known, where

$$
N_{j}(\tau)=\frac{1}{\pi} \tau^{\frac{1}{2 m}} \int_{0}^{+\infty} \rho^{-\frac{\theta}{m}}(t) \mu_{j}^{-\frac{1}{2 m}}(t) d t .
$$

Thus, we get

$$
\int_{0}^{+\infty} \frac{d N(\tau)}{\tau+t} \sim \int_{0}^{+\infty} \frac{d \tilde{N}(\tau)}{\tau+t}, \quad t \rightarrow+\infty
$$

where

$$
\widetilde{N}(\tau)=\sum_{j=1}^{n} N_{j}(\tau)
$$

Applying an appropriate tauberian theorem, we obtain the formula

$$
N(t) \sim \sum_{j=1}^{n} N_{j}(t), \quad t \rightarrow+\infty
$$

that proves Theorem 4.1.

\section{BIBLIOGRAPHY}

1. M.G. Gadoev. Spectral asymptotic of nonselfadjoint degenerate elliptic operators with singular matrix coefficients on an interval // Ufimskij Matem. Zhurn. 2011. V. 3, No. 3. P 26-54. [Ufa Math. J. 2011. V. 3, No. 3. P. 26-53.]

2. K.Kh. Boimatov. Asymptotic behavior of eigenvalues of non-self-adjoint operators // Funkts. Anal. Prilozh. 1977. V. 11, No. 4. P. 74-75. [ Funct. Anal. Appl. 1977. V. 11, No. 4. P. 305-306.]

3. K.Kh. Boimatov, A.G. Kostyuchenko. Spectral asymptotics of nonselfadjoint elliptic systems of differential operators in bounded domains // Matem. Sbornik. 1990. V. 181, No. 12. P. 1678-1693. [Math. USSR Sb. 1992. V. 71, No. 2. P. 517-531.]

4. K.Kh. Boimatov, A.G. Kostyuchenko. Distribution of eigenvalues of nonselfadjoint differential operators of second order // Vestnik MGU. Ser. I. 1990. No. 3. P. 24-31. [Mosc. Univ. Math. Bull. 1990. V. 45, No. 3. P. 26-32.]

5. G.V. Rozenblum. Spectral asymptotics of normal operators // Funkts. Anal. Prilozh. 1982. V. 16, No. 2. P. 82-83. [Funct. Anal. Appl. 1982. V. 16, No. 2. P. 145-146.]

6. G.V. Rozenblum. Conditional asymptotics for spectrum of operators close to normal // Linear and nonlinar boundary value problems. Spectral theory. Leningrad state university, Leningrad. 1986. P. 180-195. (in Russian.)

7. M.S. Agranovich and A.S. Markus On spectral properties of elliptic pseudo-differential operators far from self-adjoint ones // Zeit. Anal. Anwend. 1989. V. 8, No. 3. P. 23-260.

8. M.Sh. Birman, M.Z. Solomyak. Spectral theory of self-adjoint operators in Hilbert space // Leningrad State University, Leningrad. 1980. [D. Reidel Publ. Co., Dordrecht, 1987.]

9. G.V. Rozenblum, M.Z. Solomyak, M.A. Shubin. Spectral theory of differential operators // Itogi Nauki i Tekhniki. VINITI. Sovr. Probl. Matem. Fundament. Napravleniya. 1989. V. 64. P. 5-242.

10. M.S. Agranovich. Elliptic operators on closed manifolds // Itogi Nauki i Tekhniki. VINITI. Sovrem. Probl. Matem. Fundament. Napravleniya. 1990. V. 63. P. 5-129. 
11. M.S. Agranovich. Some asymptotic formulas for elliptic pseudodifferential operators // Funkts. Anal. Prilozh. 1987. V. 21, No. 1. P. 63-65. [Funct. Anal. Appl. 1987. V. 21, No. 1. P. 53-56.]

12. A.N. Kozhevnikov. Asymptotic behavior of the eigenvalues of elliptic systems // Funkts. Anal. Prilozh. 1977. V. 11, No. 4. P. 82-83. [Funct. Anal. Appl. 1977. V. 11, No. 4. P. 314-315.]

13. K.Kh. Boimatov. Asymptotic behavior of the spectra of second-order non-self-adjoint systems of differential operators // Matem. Zametki. 1992. V. 51, No. 4. P. 8-16. [Math. Notes. 1992. V. 51, No. 4. P. 330-337.]

14. K.Kh. Boimatov. Some spectral properties of matrix differential operators far from being selfadjoint // Funkts. Anal. Prilozh. 1995. V. 29, No. 3. P. 55-58. [Funct. Anal. Appl. 1995. V. 29, No. 3. P. 191-193.]

15. M. Faierman. An elliptic boundary problem involving an indefinite weight // Proc. Roy. Soc. of Edinburgh. 2000. V. 130A, No. 2. P. 287-305.

16. A.N. Kozhevnikov. Asymptotics of the spectrum of Douglis-Nirenberg elliptic operators on a compact manifold // Math. Nachr. 1996. V. 182, No. 1. P. 261-293.

17. S.G. Pyatkov. Riesz's bases from the eigenvectors and associated vectors of elliptic eigenvalue problems with an indefinite weight function // Siber. J. Diff. Equat. 1995. V. 1, No. 2, P. 179-196.

18. M. Sango. A spectral problem with an indefinite weight for an elliptic system // Elect. J. Diff. Equat. 1997. No. 21. P. 1-14.

19. K.Kh. Bojmatov. The generalized Dirichlet problem connected with a noncoercive bilinear form // Doklady RAN. 1993. V. 330, No. 3. P. 285-290. [Russ. Acad. Sci. Dokl. Math. 1993. V. 47, No. 3. P. 455-463.]

20. M.G. Gadoev, S.A. Iskhokov. Spectral properties of degenerate elliptic operators with matrix coefficients. // Centre de Recerca Matematica (Barcelona). Preprint series number 1078, December, 2011. 14 pages.

21. K.Kh. Boimatov. On the Abel basis property of the system of root vector-functions of degenerate elliptic differential operators with singular matrix coefficients // Sibir. Matem. Zhurn. 2006. V. 47, No. 1. P. 46-57. [Siberian Math. J. 2006. V. 47, No. 1. P. 35-44.]

22. I.C. Gohberg, M.G. Krein. Introduction to the theory of linear nonselfadjoint operators in hilbert space. Nauka, Moscow. 1965. [Amer. Math. Soc. Providence, RI. 1969.]

23. S.M. Nikol'skiur, P.I. Lizorkin, N.V. Miroshin. Weighted function spaces and their applications to the investigation of boundary value problems for degenerate elliptic equations // Izvestia VUZov. Matem. 1988. No. 8. P. 4-30. [Soviet Math. Izvestiya VUZ. Matematika. 1988. V. 32, No. 8. P. 1-40.]

24. M.G. Gadoev. On asymptotics of spectrum for a class of non-self-adjoint system // Nonclassical equations of mathematical physics. Novosibirsk: IM SB RAS. 2007. P. 78-84. (in Russian.)

Makhmadrakhim Gafurovich Gadoev,

Mirny Polytechnic Institute, a branch of Yakut State University named after M.K. Ammosov, Tikhonova str., 5/1,

678170, Mirny, Republic of Sakha (Yakutia), Russia

E-mail: gadoev@rambler.ru

Sulaimon Abunasrovich Iskhokov,

Mirny Polytechnic Institute, a branch of Yakut State University named after M.K. Ammosov, Tikhonova str., 5/1,

678170, Mirny, Republic of Sakha (Yakutia), Russia

E-mail: sulaimon@mail.ru 